\title{
The radiological investigation of urinary infections in children
}

\author{
F. STARER \\ M.R.C.P.E., F.F.R \\ Consultant Radiologist, Westminster Children's Hospital, London, S.W.1
}

\begin{abstract}
Summary
One hundred children with urinary tract infections were fully investigated radiologically. More than half showed abnormalities of the urinary tract.

It is concluded that full investigation of the urinary tract is justifiable at any age; it should be carried out in boys at the first infection, and in girls at the latest after the second infection, or if treatment did not produce the expected cure.

\section{Introduction}

Childhood urinary infections are a potent cause of adult renal failure; recent improvements in the management of chronic renal disease only serve to emphasize the need for timely prevention. It has long been recognized that urinary tract infections, particularly if recurrent or resistent to treatment are frequently associated with an abnormality of the urinary tract. Thus, Smellie et al. (1964) found structural or functional abnormalities in $50 \%$ of children with urinary tract infections. The recognition of these abnormalities is important for successful management, and depends largely on radiological investigations. These consist in the main of excretion pyelography and micturating cystography. Stansfeld (1966) has estimated the incidence of urinary infections in the general population to be $0 \cdot 17 / 1000$ boys and $0 \cdot 4 / 1000$ girls. The investigation of such large numbers provides X-ray departments with a great deal of work, and presents a considerable radiation burden to the population. We, therefore, thought it would be of interest to review our own findings in order to assess whether the expenditure of time, effort and radiation was worthwhile.

\section{The series}

For this purpose the records of 100 children with urinary infections who had been fully investigated radiologically were reviewed. Criteria for diagnosis were strictly observed, and correspond to those described by Scott (1968). Ages ranged from birth to 15 years (Fig. 1). The 100 records which were picked at random from the material available in the $\mathrm{X}$-ray department were unselected, but clearly
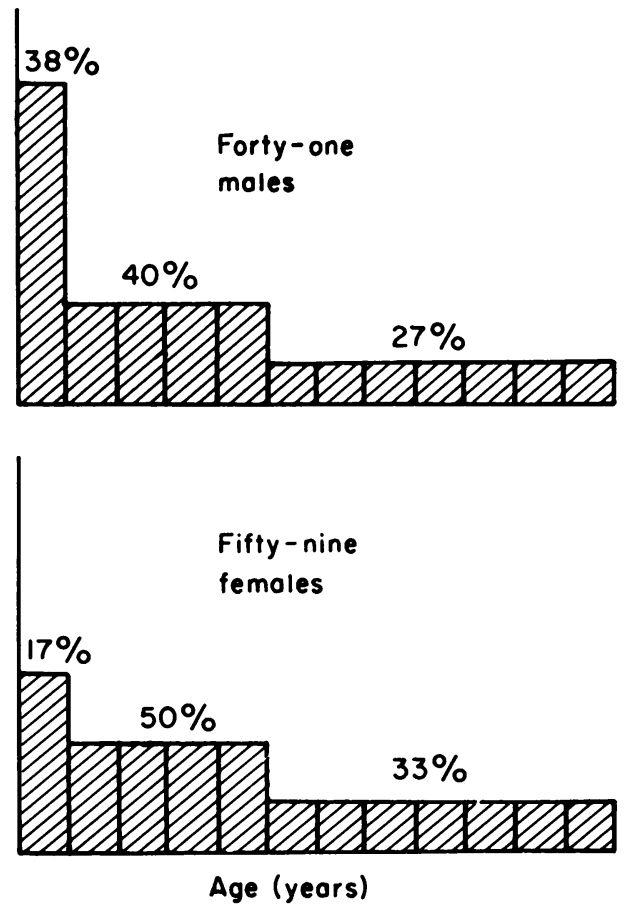

Fig. 1. Age distribution of 100 children investigated for urinary infections.

considerable selection had occurred before the child came to us because the patient had first to be referred by his general practitioner to the hospital, and then by the hospital consultant to the X-ray department. Our policy has been to carry out pyelograms on all; cystograms are performed on all boys and on any girls in whom primary treatment was unsuccessful, or who show any pyelographic abnormality. With increasing experience, there has been a tendency to widen the indications for cystography, so that more and more of these examinations are being carried out. All children in this series had both examinations, and this would tend to weight this series somewhat with a higher incidence of anatomical abnormalities compared with a completely random series. 


\section{Techniques}

Standard, well-known techniques were used, but a few comments may be apposite.

\section{Pyelography}

Pyelography was carried out after clearing the gut and under only mild dehydration. A minimum of $20 \mathrm{ml}$ of contrast medium was used, even for neonates, always given intravenously. Intramuscular injection should be avoided. Since visualization of renal outlines is very important we have never hesitated to use tomography to show the kidneys. A film taken with the tube moving through a small arc adds little radiation to the examination, and may on the contrary save a number of useless exposures. Small children must be well sedated for this examination.

\section{Micturating cystography}

This was done under basal sedation; I consider the use of general anaesthesia advocated by some authorities to be nearly always unnecessary and undesirable. The urethral catheter is passed under strictly aseptic conditions, and infection from it has not been a problem. Routine antibiotic cover was not used. The bladder was viewed by image intensification and television monitor, and the image recorded on a videotape recorder. The latter has advantages over cine film: it can be viewed immediately and does not require any increase in radiation. The catheter was connected by a drip-set to a bottle containing $20 \%$ diodone as described by Trapnell (1965), thereby maintaining complete sterility. One other requirement is a plentiful supply of patience, particularly in older children who cannot always micturate to order.

\section{Results}

Table 1 summarizes the results found in 100 children.

TABLE 1

\begin{tabular}{lr}
\hline Hydronephrosis & 25 \\
Atrophic pyelonephritis & 8 \\
Bladder diverticula & 8 \\
Bladder residue & 8 \\
Ureterocoele & 2 \\
Calculi & 2 \\
Duplex kidney & 5 \\
Horse-shoe & 1 \\
Ureteric reflux & 43 \\
\hline
\end{tabular}

In some children more than one abnormality was discovered, and there were only thirty-five children who appeared normal in all respects: nine males and twenty-six females.

Several of these abnormalities require further discussion.

\section{Hydronephrosis}

The causes of hydronephrosis can be further broken down as shown in Table 2 .

\begin{tabular}{lr}
\multicolumn{2}{c}{ TABLE 2 } \\
\hline Pelvi-ureteric obstruction (7) \\
Cause unknown & 5 \\
Abnormal vessels & 2 \\
Ureterocoele & 2 \\
Pin-hole ureteric orifice & 1 \\
Urethral valve & 1 \\
Exomphalos & 1 \\
Horse-shoe kidney & 1 \\
Reflux & 12 \\
Total & 25 \\
\hline
\end{tabular}

It will be seen that the commonest single cause of hydronephrosis is vesico-ureteric reflux. Reflux as a common cause of hydronephrosis has been recognized only fairly recently, but the finding accords with other observers. For instance Shopfner (1965), in a review of 300 children with urinary infections, found that dilatation of the pelvi-calycine system was more often due to reflux than to pelvi-ureteric obstruction. He found that obstruction had been diagnosed in eighty-three children and led to seventyfour operations in 1953. Ten years later, after the importance of reflux had been recognized, obstruction was diagnosed only five times during a period of 1 year, and all were operated upon. The mechanism whereby reflux causes hydronephrosis is not fully established. The degree of pelvic dilatation caused by reflux into the pelvis is quite astonishing, and it may be that the constant stretching of the elastic tissue in the pelvis finally leads to its permanent distention. Other suggestions have been put forward. Hodson \& Edwards (1960) propose vascular compression as a factor, while Hutch, Hinman \& Miller (1962) believe that distention of the pelvis may cause kinking at the pelvi-ureteric junction with ultimately permanent hydronephrosis. No doubt infection may also play a part, but we have evidence amongst our own material (but not included in this series) that infection is not an essential factor in the development of hydronephrosis.

Next in frequency was obstruction at the pelviureteric junction. This should be diagnosed only in the absence of reflux, and if stasis in the pelvis can be demonstrated by a retrograde pyelogram. (This is one of the few indications for performing this examination. Improved excretion pyelography has made it largely unnecessary, and it is liable to cause oedema of the ureteral mucosa with possible consequent infection.) In patients in whom no cause can be demonstrated radiographically, one is dealing either with a valvular mechanism at the junction, or some abnormality in the dynamics of the upper ureter. An important factor in the flow of urine is 
normal peristaltic activity of the pelvis and ureter. Urine will not flow, even in the absence of obstruction, if peristalsis is absent or abnormal. (Good examples of this are patients with peri-ureteric fibrosis, who develop severe hydronephrosis in the presence of a perfectly patent but rigid and aperistaltic ureter.) It is highly probable that the hydronephrosis sometimes associated with duplex or horse-shoe kidneys also depends on abnormal pelviureteric dynamics.

It will be noted that abnormal vessels are thought to have been the cause of obstruction in two patients. At one time this was a widely accepted cause of hydronephrosis, but it has lately received much more critical consideration. More often than not a vessel running close to a dilated renal pelvis had no part in the genesis of the hydronephrosis, but our evidence suggests that occasionally the vessel is responsible. In these two patients the hydronephrosis was relieved by division of the responsible aberrant vessel; such a vessel is easily demonstrated by aortography preoperatively. This examination, using the Seldinger technique of percutaneous femoral catheterization, can be carried out at any age from birth. There should be good spatial correlation between the aberrant vessel and the point of obstruction before the obstructing role of the vessel is accepted. Obstruction to single calyces may also be caused by an arterial branch within the renal substance, or less commonly by a vein accompanying an artery (Fraley, 1966; Starer, 1968). Although several such cases have been seen in our department, none happen to be included in this series.

Another lesion in this group requiring special comment is urethral valve (Fig. 2). It is usually stated that these children present with a poor urinary stream, but this is by no means always the case, or the fact is not recognized by the mother. Infection in an obstructed urinary system may then be the first sign. By this time damage to the kidneys is considerable, and in fact the kidneys are usually already damaged at birth from the effects of the valve before birth. The prognosis is surprisingly poor, and the condition is best recognized during a micturating cystogram. This also frequently reveals the presence of reflux.

Ureterocoeles, caused by prolapse of the lower ureteral mucosa into the bladder were observed twice. Often they develop in association with an ectopic ureter, but this happened not to have been the case in these two children. When small, they present a rather characteristic 'halo' or 'cobra-head' appearance which can easily be missed on the IVP. When large, they can be mistaken for gas in the rectum. In the course of their growth they cause obstruction, first to their own ureter (Fig. 3) and ultimately they may even obstruct the opposite ureter. Another problem concerning ureterocoeles

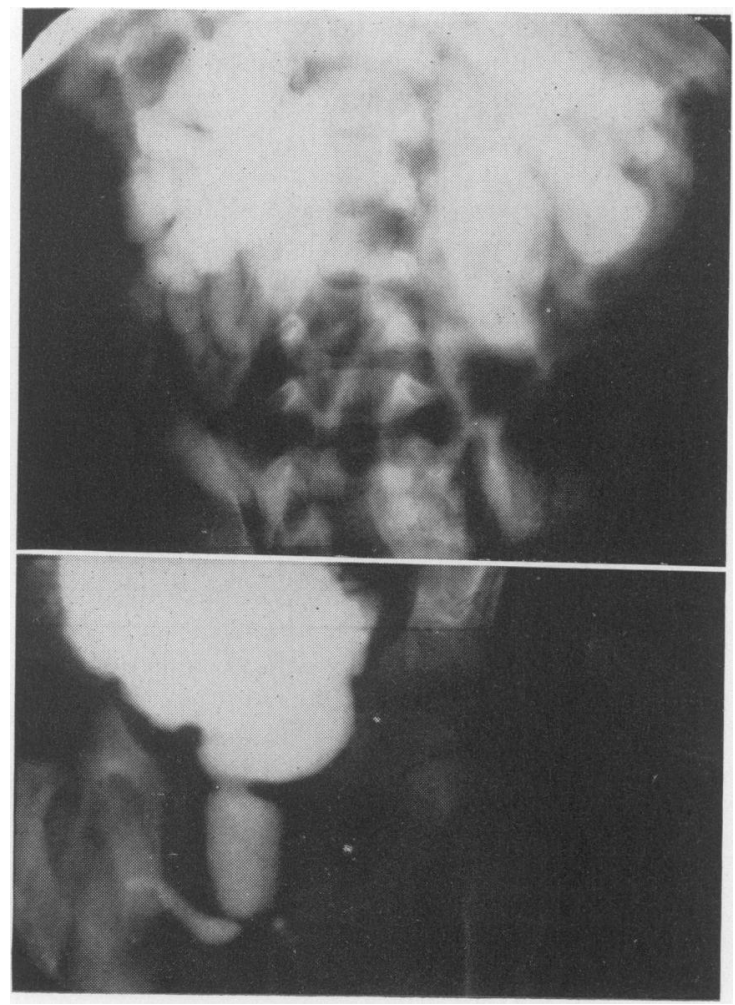

FIg. 2. Posterior urethral valve and its effect on the kidneys: there is gross bilateral hydronephrosis.

arises when an ectopic ureter is obstructed, with loss of function of part of a kidney, most usually the upper moiety. The combination of an upper renal mass with an incomplete set of calyces should provide the diagnosis on the pyelogram.

\section{Bladder diverticula}

These were found in eight patients in this series. Diverticula are rarely able to empty themselves completely. Indeed, one can often observe a diverticulum becoming more distended as the bladder contracts during micturition. Once the urine has become infected, the diverticulum provides a constant reservoir of infection. The size of the diverticulum matters little-tiny ones are just as potent in maintaining infection as large ones. Most bladder diverticula can be seen during the course of a pyelogram, particularly if oblique views are taken. Surprisingly, however, one occasionally detects diverticula during a micturating cystogram which were not seen on the pyelogram (Fig. 4). Small diverticula can also quite easily be missed during cystoscopy. One source of error must be kept in mind: a half-empty bladder may show a trabeculated 
outline which could be mistaken for numerous small diverticula. The most reliable method for the diagnosis of diverticula is cystography.

\section{Bladder residue}

The significance of a bladder residue after micturition is by no means clear. Theoretically a bladder which never empties completely would be capable of maintaining infection in exactly the same way as a bladder diverticulum. The difficulty lies in assessing its significance on a single examination carried out under highly abnormal circumstances. It is probably better assessed in the post-voiding film at the end of an excretion pyelogram than after cystography. Most children under these circumstances empty completely, and it is our impression that incomplete bladder emptying is more common in children with urinary tract infection. In the absence of a matched control group of children without infection this cannot be more than just an impression at the moment.

\section{Ureteric reflux}

This represents the largest group of children in this series. The dire significance of reflux in the perpetuation of infection is now widely recognized. Even without infection reflux, if severe, may occasionally affect the kidney, but in the vast majority of cases it is infection which does most of the damage. Pathogenic organisms are found in the urine from time-to-time, but the urinary tract is a self-cleaning and self-sterilizing system provided

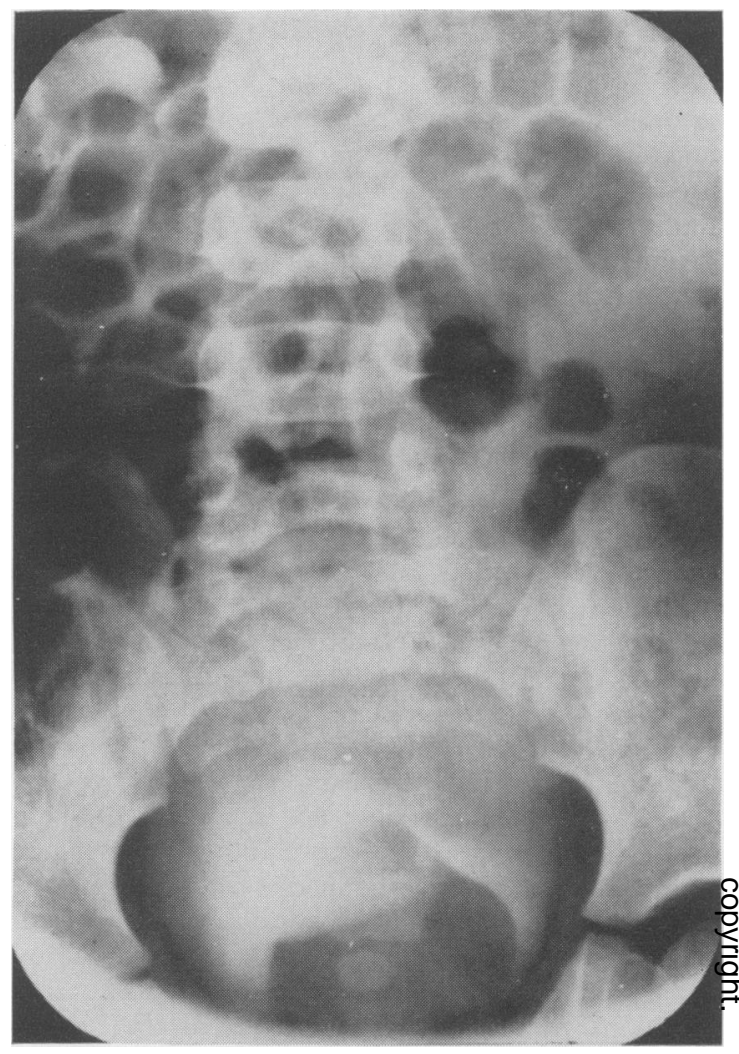

Fig. 3. Large ureterocoele causing hydronephrosis. it can be completely and continuously flushed. Like

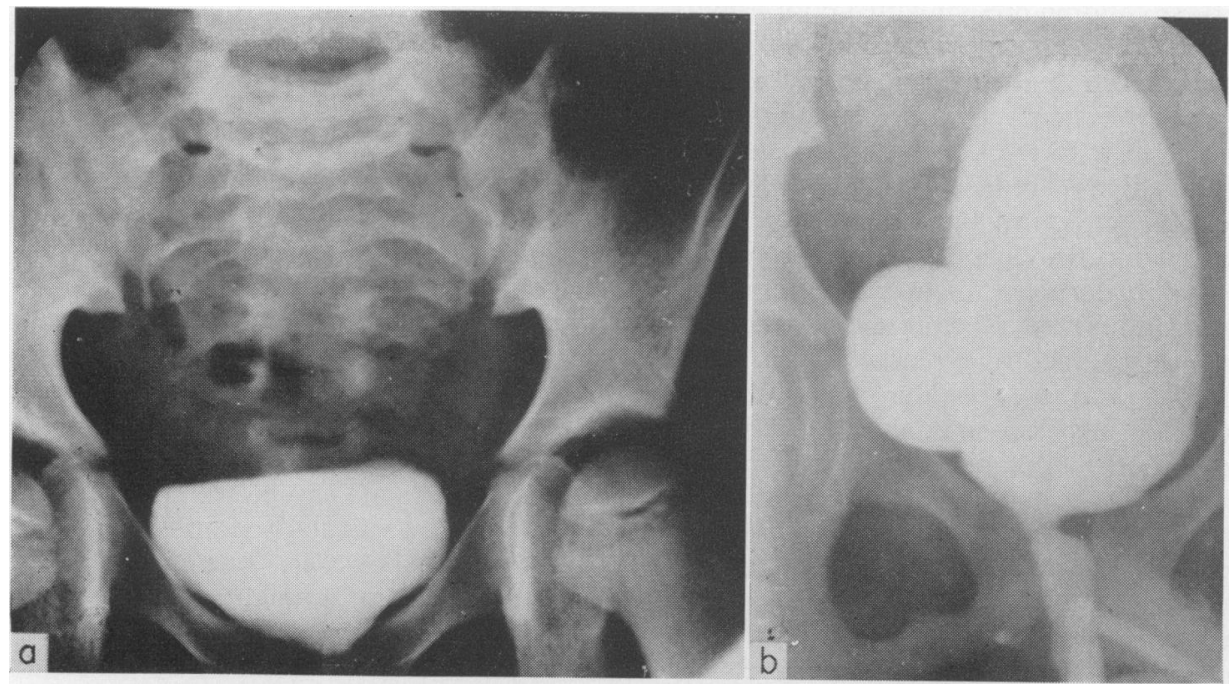

Fig. 4. Diverticulum of bladder, demonstrated only by micturating cystogram. (a) Pyelogram, (b) cystogram. 
bladder diverticula, reflux prevents complete emptying and provides a constant source of re-infection. This is very strikingly illustrated when watching fairly severe reflux: as the bladder empties, the ureter and renal pelvis fill. The refluxed urine gradually refills the bladder, only to reflux at the next act of micturition. This incidentally explains why these patients sometimes feel an urge for two or more acts of voiding over a brief period of time.

It is now well established that reflux is abnormal at any age (Gibson, 1949; Iannaccone \& Pazironi, 1955; Jones \& Headstream, 1958; Leadbetter, Duxbury \& Dreyfuss, 1960; Lich et al., 1964). Iannaccone (1966) found transient reflux very rarely in very small normal babies, and this has also been our experience, but as a rule it should be regarded as an abnormal finding.

The cause of reflux is not clearly established. Normally it is prevented by the valve-like insertion of the ureter into the bladder. This insertion is much shorter in infants than in adults, and reflux is most probably due to an abnormal insertion of the ureter into the bladder. Often this can be observed cystoscopically. The picture is confused by the very frequent association with infection, and there is a tendency to blame the oedema and rigidity of the terminal ureter and bladder caused by infection for the reflux. There is some experimental support for this view, moreover, since reflux can be produced in dogs by inducing a severe, protracted urinary infection. Despite this it is more likely that reflux is a primary event of developmental origin which can most certainly be aggravated by severe urinary infection. A further observation supporting the developmental origin of reflux is the fact that it is much commoner in children than in adults. Baker et al. (1966) found reflux in $26.4 \%$ of their paediatric urological patients, but in only $5 \cdot 2 \%$ of adult patients under similar circumstances. This would seem to be due to growth of the intravesical portion of the ureter. It also demonstrates that reflux may cease despite the presence of continuing infection.

A problem which in the past has caused much confusion is the presence of bladder-neck obstruction in patients with reflux. Some years ago it was commonly held 'no reflux without bladder-neck narrowing'. The situation is well illustrated by the claims of Stewart (1961) who diagnosed obstruction in $95 \%$ of patients with reflux; Leadbetter (1961) who found it in 50\% and Hutch (1961) who stated it was 'uncommon'. It is now generally held that bladder-neck obstruction is indeed uncommon, and that its frequent diagnosis on micturating cystograms in the past was based on an incomplete knowledge of the varying appearances of the normal bladderneck. This statement is equally true of male and female patients. It is probable that the diagnosis of meatal stenosis popular in some departments is similarly untenable. No cases of bladder-neck or meatal stenosis could be recognized in this series. In the present state of knowledge it is doubtful if we would accept such a diagnosis without careful cystometric and endoscopic confirmation. An excellent account of the value of flow measurements in this problem is given by Scott (1968). It must be added the cystometry has not been a very rewarding tool in clinical practice in our hands.

With this as background we may now turn to a closer analysis of the forty-three children with reflux in the series. Reflux was observed in $75 \%$ of patients below 1 year of age, but in only $33 \%$ of patients above 1 year. Allowing for their age distribution, there was no difference in the incidence between boys or girls, nor was there any sex difference in the severity of the reflux. The two sides were equally affected. All the patients with pyelonephritis showed reflux on the affected side. The effect of reflux on the development of pyelonephritis is neatly illustrated by patients with complete double ureters on one side of which only one ureter was affected by reflux. Invariably it was the part of the kidney drained by the abnormal ureter which developed pyelonephritis. Although it is usually stated that reflux is more common into the upper moiety our experience has suggested that there is little difference between the upper and lower parts in this respect (Figs. 5 and 6).

Reflux disappeared in seven patients. In six the reflux was regarded as mild when first seen and in one as moderate. We have not observed disappearance of really severe reflux. It should be remembered, however, that even in the same child reflux is a somewhat variable event. At its most severe, contrast medium may flood back into the kidney as soon as it is introduced into the bladder. On the other hand, reflux may be slight and quite intermittent. It is then liable to be missed unless the ureters are observed continuously during filling of the bladder-imposing an intolerable radiation burden on the patient. Screening, therefore, must be intermittent, and occasionally a whiff of reflux will be missed. Finally reflux may occur only during the phase of bladder emptying.

In only twenty-six of these forty-three patients was the IVP abnormal and would have indicated the need for micturating cystography; the rest had entirely normal pyelograms. The abnormalities consisted of varying degrees of calyceal and pelvic dilatation with or without parenchymal scarring, and sometimes dilatation of the ureter involved. In this case it is usually the lower third which is mainly affected. Unfortunately a normal IVP is not. a safe indication that a cystogram is unnecessary. The difference in the appearances of the kidneys between the pyelograms and cystogram can be quite startling. A normal- 


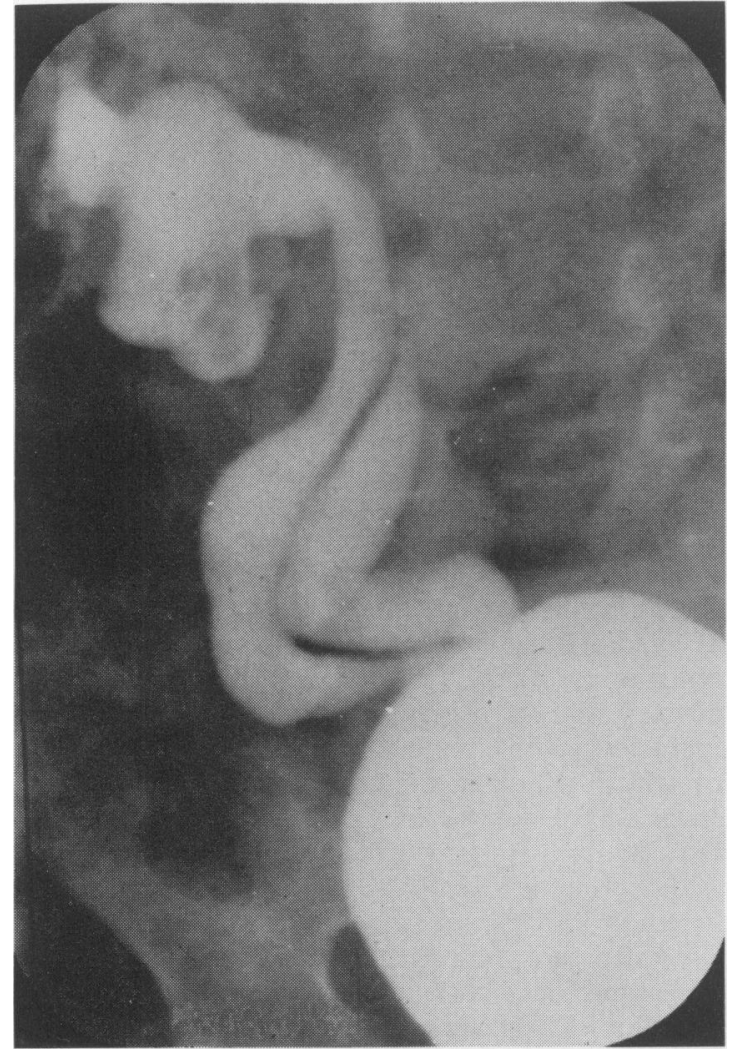

FIG. 5. Micturating cystogram-reflux up bifid right ureter.

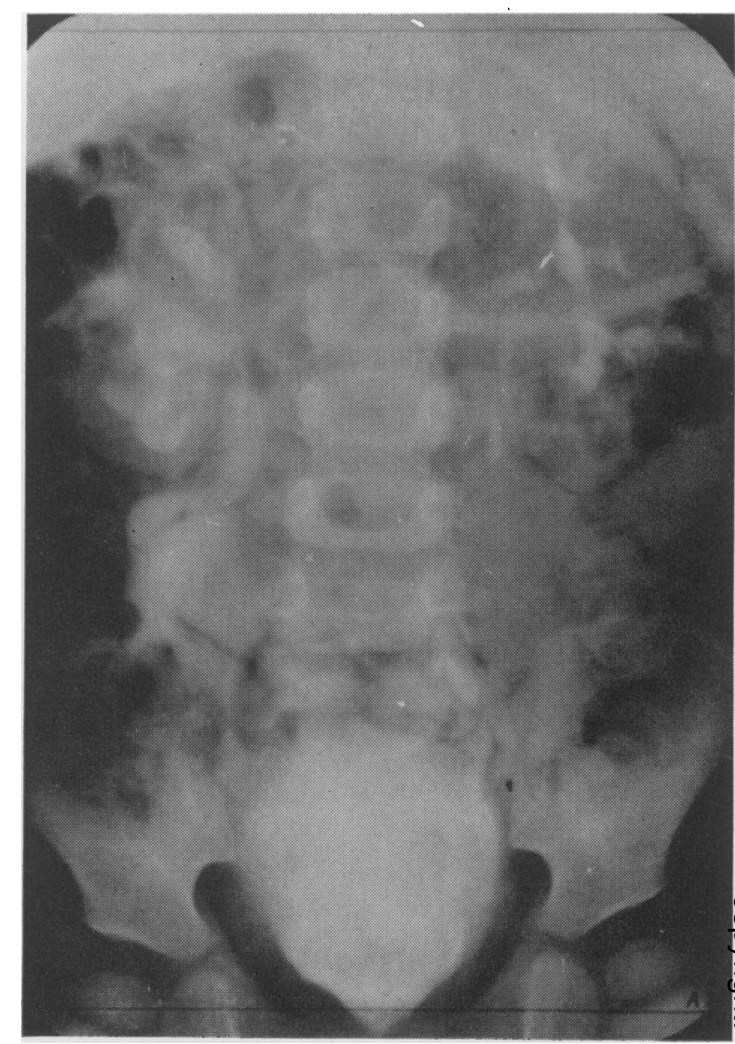

FIG. 6. Pyelogram of same patient.
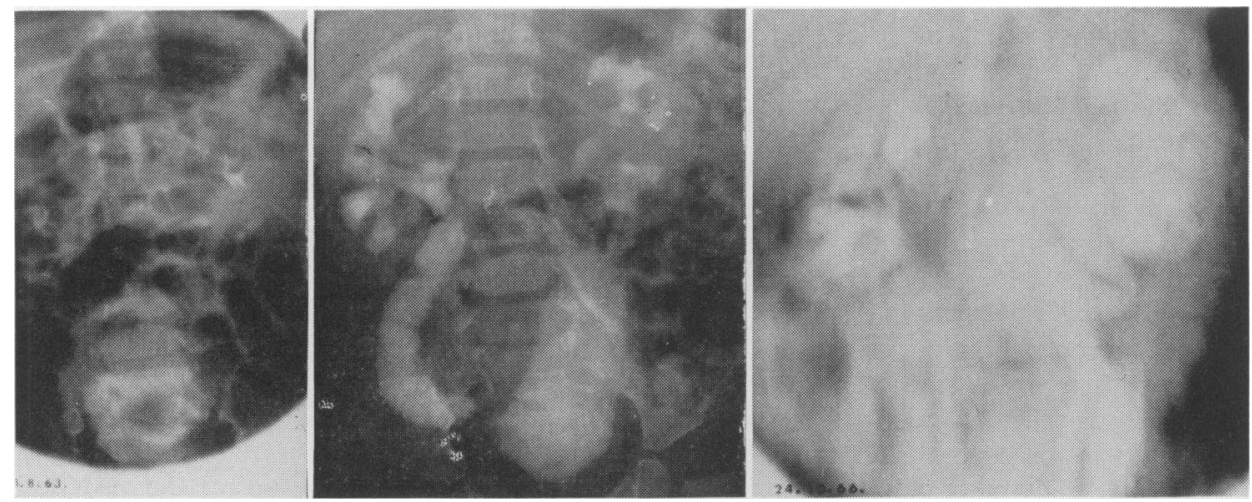

FIG. 7. Bilateral reflux with pyelonephritis.

looking pelvis and ureter may become grossly distended during bladder filling.

A further aspect that merits observation and comment is the behaviour of the ureter. A normal ureter shows orderly peristalsis, whereas dilated, refluxing ureters often exhibit irregular segmentation and disorganized or reversed peristalsis. Finally peristalsis ceases altogether and the ureter becomes an inert tube. This abnormality of ureteric motility is no doubt yet a further factor in causing hydronephrosis. It also raises the possibility that in some patients the mechanism of reflux may be more complex than simply valvular incompetence at the uretero-vesical junction. Lack of ureteric peristalsis is of importance 


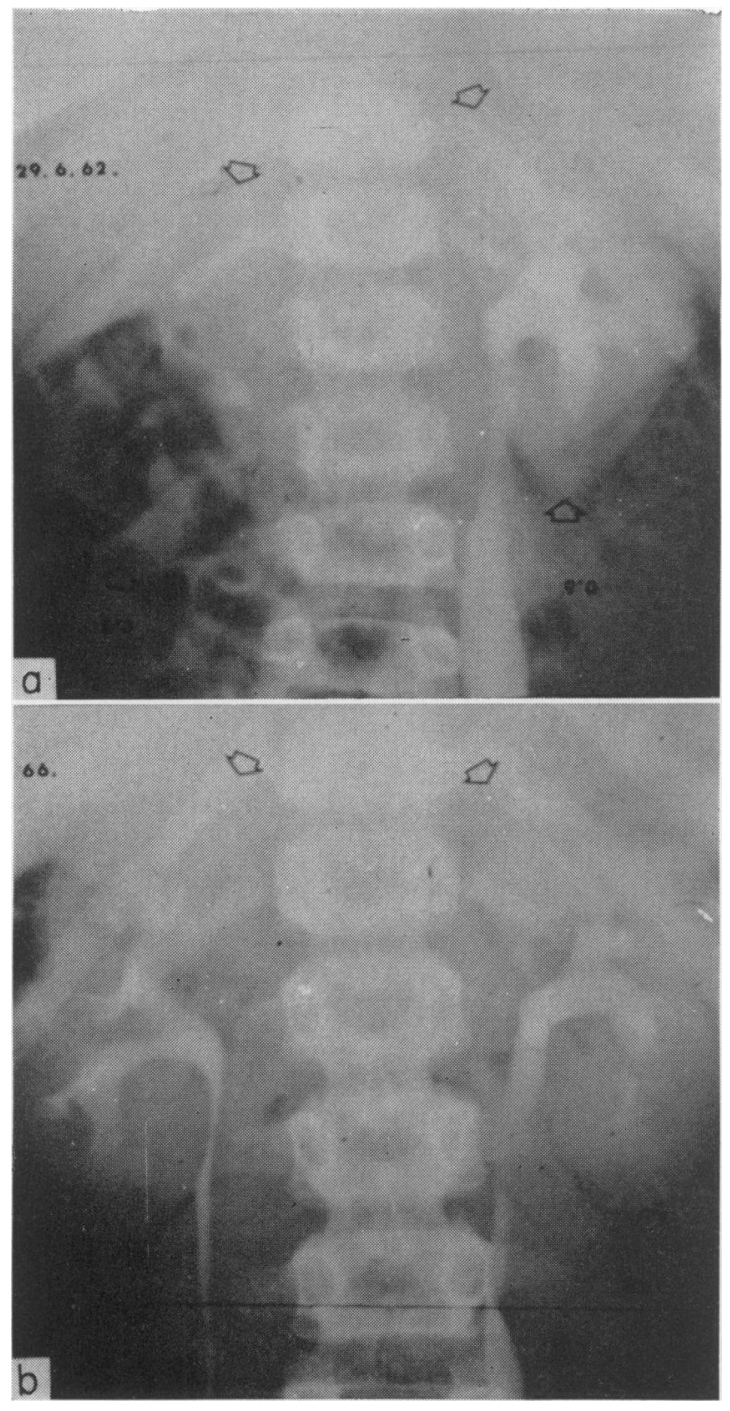

FIG. 8. Left-sided reflux. The right kidney is growing normally, the left side has failed to grow. (a) 29 June 1962: left kidney $9.0 \mathrm{~cm}$, right kidney $8.3 \mathrm{~cm}$; (b) 3 June, 1966: left kidney $9.3 \mathrm{~cm}$, right kidney $10.3 \mathrm{~cm}$.

because it reduces the success rate of ureteric implants. We have seen patients, however, in whom a successful re-implant was carried out despite the presence of a paralysed ureter, with some recovery of ureteric activity after the operation.

The position as regards reflux may, therefore, be summarized as follows:

(1) For practical purposes reflux is always abnormal.

(2) If not very severe, reflux may cease spontaneously despite the presence of infection, but is more likely to do so if infection is controlled.
(3) Reflux is the most important single factor in maintaining infection and causing chronic pyelonephritis. In turn it is probably aggravated by infection.

(4) Reflux cannot be reliably predicted from the appearances of the excretion pyelogram.

(5) Bladder-neck or urethral obstructions are very rare events with the exception of posterior urethral valves.

\section{Pyelonephritis}

Atrophic pyelonephritis was already established in eight children when they first presented for investigation. In these, much damage had already been done but it is to be hoped that the course of the disease can be slowed by treatment. The radiological features of pyelonephritis are now well known. They consist of irregularities and blunting of the calyces and irregularities of the cortical outlines (Figs. 7 and 8). The latter is often a more sensitive index of renal damage than changes in the calyces, and this is one reason why demonstration of the renal outline is so important. Cortical irregularities due to pyelonephritic scarring must be distinguished from foetal lobulation. Pyelonephritic scars are found opposite renal calyces, whereas the depressions of foetal lobulation occur between calyces. In practice the distinction is not as easy as textbooks would lead one to expect. Another important feature of active pyelonephritis is that it prevents normal growth of the affected kidney. Therefore, failure of a kidney to grow, even in the absence of other changes, is an ominous sign of continuing renal damage. The second reason for showing the renal outlines is to enable the radiologist to obtain serial measurements over a period of months or years. It must be admitted that pyelonephritis can exist in the absence of any pyelographic changes.

Pyelograms of diagnostic quality can be obtained in children with renal failure in the presence of a raised blood urea. It is necessary to increase the amount of contrast medium by two or three times, and tomography is very valuable in this situation also.

\section{Conclusion}

The high incidence of abnormalities found in this series justifies full radiological investigation of the urinary tract (pyelography and cystography) in patients with urinary infections. We suggest that all boys with urinary infections be investigated during the first episode; girls, more liable to these infections, require investigation if there has been more than one episode of infection, or if treatment does not have the expected result. However, close observation over a number of years following apparently successful treatment is essential. Preuss 
et al. (1969) have recently published an interesting follow-up on a large series of children with urinary infections. One hundred and eighty-six children were recalled from 2 to 9 years following apparently successful treatment. It was found that $69.4 \%$ were well, but $30.6 \%$ had developed some renal damage, and $16.1 \%$ were in need of urgent treatment. Further breakdown of the series showed that amongst children apparently cured by a single course of treatment, $75.7 \%$ were well but $24.3 \%$ had not fully cleared up and showed residual damage, and if several courses of treatment had been required, $44.2 \%$ were abnormal on follow-up and $25.6 \%$ needed immediate further treatment. This only serves to emphasize the urgent need for full investigation, treatment and follow-up of these patients.

\section{Acknowledgments}

My thanks are due to the Department of Medical Photography, Westminster Hospital, for the illustrations. I am grateful to my clinical colleagues for their help, and for the many stimulating discussions we have had. Finally, I should like to express my gratitude to the Agnes Spencer Trust for the video tape recorder which has been most valuable.

\section{References}

Baker, R., Maxted, W., Maylath, J. \& Schuman, I. (1966) Relation of age, sex and infection to reflux: Data indicating high spontaneous cure rate in paediatric patients. Journal of Urology, 95, 27.

Fraley, E.E. (1966) Vascular obstruction of superior infundibulum causing nephralgia. New England Journal of Medicine, 275, 1403.

GiBSON, H.M. (1949) Ureteral reflux in the normal child. Journal of Urology, 62, 40.
Hodson, C.J. \& Edwards, D. (1960) Chronic pyelonephritis and vesico-ureteric reflux. Clinical Radiology, 11, 219.

Huтch, J.A. (1961) Bladder vasculature with special reference to the ureterovesical junction. Journal of Urology, 85, 119.

Hutch, J.A., Hinman, F. \& Miller, E.R. (1962) Reflux as a cause of hydronephrosis and chronic pyelonephritis. Journal of Urology, 88, 169.

IANNACCONE, G. (1966) Ureteral reflux in normal infants. Annales de radiologie, 9, 31.

Iannaccone, G. \& Pazironi, P.E. (1955) Ureteral reflux in normal infants. Acta radiologica, 44, 451.

JoNes, B.W. \& Headstream, J.W. (1958) Vesicoureteral reflux in children. Journal of Urology, 80, 114.

LEADBETTER, G.W. (1961) Following panel discussion on 'Ureteral reflux in children'. Journal of Urology, 85, 119.

LeadbetTer, G.W., Duxbury, J.H. \& Dreyfuss, J.R. (1960) Absence of vesicoureteral reflux in normal adult males. Journal of Urology, 84, 69.

Lich, R., Howerton, L.W., Goode, L.S. \& Davis, L.A. (1964) The ureterovesical junction of the newborn. Journal of Urology, 92, 436.

Preuss, H.J., Deckart, H., Breunung, M. \& Padlet, H. (1969) Röntgenologische, nuklearmedizinische und klinische Ergebnisse von Nachuntersuchungen der Harnweg Infektion im Kindesalter. Fortschritte Auf Dem Gebiete Der Röntgenstrahlen, 110, 462.

ScotT, J.E.S. (1968) Paediatric Urology (Ed. by D. Innes Williams). Butterworth, London.

ShopfNer, C.E. (1965) Changing concepts of paediatric uroradiology. Journal Lancet, 85, 525.

Smellie, J.M., Hodson, C.J., Edwards, D. \& Normand, I.C.S. (1964) Clinical and radiological features of urinary infection in childhood. British Medical Journal, 2, 1222.

STANSFELd, J.M. (1966) Clinical observations relating to incidence and aetiology of urinary-tract infections in children. British Medical Journal, 1, 631.

STARER, F. (1968) Partial hydronephrosis due to pressure from normal renal arteries. British Medical Journal, 1, 98.

STEWART, C.M. (1961) Following panel discussion on 'Ureteral reflux in children'. Journal of Urology, 85, 119.

TRAPNELL, D.H. (1965) A simple, sterile method of filling the bladder. British Journal of Radiology, 38, 553. 